\title{
Nordiques
}

35 | 2018

Tove Jansson : Par delà les genres. Pratiques

linguistiques dans le Norden du XXIe siècle : Quels

enjeux sociaux?

\section{Écologie des langues dans le Norden}

\author{
Karl Erland Gadelii
}

\section{CpenEdition}

Journals

Édition électronique

URL : https://journals.openedition.org/nordiques/1570

ISSN : 2777-8479

Éditeur :

Association Norden, Bibliothèque de Caen la mer

Édition imprimée

Date de publication : 1 mai 2018

Pagination : 77-93

ISBN : 979-1-0959140-1-3

ISSN : 1761-7677

\section{Référence électronique}

Karl Erland Gadelii, «Écologie des langues dans le Norden », Nordiques [En ligne], 35 | 2018, mis en ligne le 02 février 2021, consulté le 06 octobre 2021. URL : http://journals.openedition.org/nordiques/ 1570 


\section{Écologie des langues dans le Norden}

Karl Erland Gadelii*

\section{RÉSUMÉ}

Cet article examine la situation linguistique du Norden du point de vue de l'écologie des langues dans le Nord. Après un aperçu du concept de Norden comme communauté linguistique, la question sera posée de l'idéalisation de cette région, où chaque pays est imaginé comme linguistiquement homogène, et où la communication panscandinave est facilement assurée grâce à une intercompréhension considérable. Cet article met en lumière les conflits linguistiques dans ces régions, particulièrement dans les (ex-)colonies du Danemark et de la Suède. Enfin, il ouvre la réflexion sur les critères qui permettent d'expliquer pourquoi une langue (ex-)coloniale simpose (tel le suédois au Sápmi) ou non (comme le danois aux îles Féroé).

\section{ABSTRACT}

This article examines the linguistic situation in the Nordic countries from the viewpoint of the ecology of languages in the North. Starting out with a presentation of the idea of the Nordic countries as a linguistic community, it then questions the fairly idyllic image of the North where every country is supposed to be linguistically homogeneous, and where pan-Scandinavian communication is guaranteed by an important mutual intelligibility between languages involved. The author rather holds that all Nordic regions host more or less serious linguistic conflicts, in particular the Nordic (ex)colonies, i.e. all Nordic territories except Denmark and Sweden, once colonized by the latter. The article gives an overall picture of linguistic "hegemony" in the Nordic countries, and asks what criteria may decide whether an (ex-)colonial language imposes itself (as in the case of Swedish in Sápmi) or not (see Danish in the Faroe Islands).

${ }^{*}$ Karl Erland Gadelii est professeur de linguistique nordique à l'université Paris-Sorbonne (Paris IV). Il s'intéresse à la morphosyntaxe nordique comparative, mais également aux contacts des langues dans l'espace nordique et à la politique linguistique. 


\section{INTRODUCTION}

Il existe plusieurs organismes dont le but est de promouvoir l'idée du Norden comme une communauté historique, politique, culturelle et linguistique homogène. Mentionnons Nordiska rådet, Nordiska ministerrådet för Kultur, Nordiska ministerrådet för Utbildning och Forskning, Föreningen Norden, Nordisk Sprogråd et Kulturkontakt Nord ${ }^{1}$.

La volonté politique de présenter le Norden comme une aire homogène ne manque donc pas, mais reflète-t-elle la réalité ? Les citoyens des pays nordiques se sentent-ils membres d'une grande communauté linguistique ? À propos de la notion de "communauté linguistique ", renvoyons aux travaux de Olle Josephson $^{2}$. Citant Joshua Fishman ${ }^{3}$, Josephson évoque quatre critères cruciaux pour qu'on puisse parler d'une communauté linguistique :

1. similitude entre les langues concernées ;

2. interaction entre les locuteurs des différentes langues ;

3. adhérence des locuteurs des différentes langues aux mêmes attitudes linguistiques, plus un sentiment d'être membres d'une communauté linguistique superposée (même s'il s'agit d'une " communauté imaginée ") ;

4. politique linguistique commune.

Nous démontrerons dans cet article que ces quatre critères sont bien remplis dans le cas du Norden, lequel constitue donc une communauté linguistique solide.

Commençons par le dernier critère, la politique linguistique commune, qui est bien respecté. Le grand nombre d'organisations pan-nordiques que nous venons d'énumérer en témoigne, car elles ont toutes comme objectif de promouvoir la coopération nordique. En politique linguistique, c'est plus spécifiquement l'organisation Nordisk Sprogråd (Conseil des langues nordiques) qui s'en charge.

Quant au troisième critère, on peut d'abord constater que le Norden n'a pas connu de "guerres linguistiques " du même type que celles observées en Belgique ou au Canada ${ }^{4}$. En outre, Olle Josephson ${ }^{5}$ considère que les différences entre les pays nordiques en termes de politique linguistique ne sont pas drastiques, mais il mentionne quand même quelques spécificités :

- le purisme islandais ;

- le laxisme suédois ;

1 En français, respectivement le Conseil nordique, le Conseil des ministres nordiques pour la culture, le Conseil des ministres nordiques pour l'éducation et la recherche, l'Association nordique, le Conseil des langues nordiques et Contacts de cultures dans le Nord.

2 Olle Josephson, «La communauté linguistique nordique. Éléments de comparaison des politiques linguistiques des pays scandinaves ", Nordiques, n 24, 2012, p. 15-35.

3 Ibid., p. 17.

4 Voir par exemple Michel de Cóster, Les enjeux des conflits linguistiques. Le français à l'épreuve des modèles belge, suisse et canadien, Paris, L'Harmattan, 2007.

5 Olle Josephson, op. cit., p. 30-32. 
- le conflit entre bokmål (dano-norvégien) et nynorsk (néo-norvégien) en Norvège ; - les droits des finlandssvenskar (suédophones en Finlande);

- la "guerre de la mayonnaise " au Danemark (= la question de choisir une orthographe savante ou populaire).

Parmi les éléments de cette liste, les conflits liés à la Suède et au Danemark (le laxisme et la "guerre de la mayonnaise ") semblent donc minimes par rapport aux situations des autres pays nordiques. À partir de ce présupposé, Olle Josephson précise que « l'établissement d'une langue nationale [au Danemark et en Suède] n’a jamais été lié à un mouvement de libération nationale, comme ce fut le cas avec le norvégien en Norvège, le finnois en Finlande [et l'islandais en Islande] $»^{6}$. Josephson poursuit en soutenant que « le danois a aussi revêtu une importance symbolique considérable lorsque l'identité danoise a été redéfinie après la défaite contre la Prusse en 1864. La relation entre le danois et l'allemand au Jutland du Sud était très tendue jusqu'en $1920 \%$.

Le caractère non conflictuel de la politique linguistique en Suède découle selon Josephson du fait que la Suède n'a pas participé à la seconde guerre mondiale ; et, par conséquent, « les autorités linguistiques ont prôné en Suède la norme fonctionnelle ou le principe de l'utilitarisme [...]. Le choix de langue, les néologismes et les conventions orthographiques sont évalués non par rapport à leurs racines historiques ou à leur valeur identitaire, mais par rapport à leur fonctionnalité dans la vie quotidienne ».

Cette attitude suédoise est caractérisée par Josephson de « confiance naïve dans la norme fonctionnelle ».

Tous ces éléments indiquent donc que le troisième critère s'applique à la communauté du Norden. Nous reviendrons par ailleurs sur le fait qu'il repose sur un esprit de consensus et de tolérance qui peut être mis en cause. En tant que suédophone, nous constatons nous-même une appartenance très forte à une communauté linguistique superposée : quand nous, les Nordiques, nous croisons à l'extérieur du Norden, nous parlons normalement nos langues nordiques respectives et nous nous considérons comme des compatriotes.

Quant au deuxième critère, l'interaction entre les locuteurs des différentes langues, nous rejoignons encore une fois Olle Josephson ${ }^{7}$ lorsqu'il constate que les Nordiques n'ont jamais autant voyagé et travaillé dans les pays nordiques avoisinants qu'aujourd'hui ${ }^{8}$.

Le premier critère énuméré ci-dessus concerne la similitude entre les langues concernées, laquelle est à son tour liée à l'intercompréhension. Sur ce point, les langues

6 Ibid., p. 31.

7 Ibid., p. 33.

8 Pensons à la conurbation d'Øresund, vrai centre économique où se côtoient Danois et Suédois dans leurs lieux de travail. Mentionnons également le fait que de nombreux Suédois s'orientent vers la Norvège, où l'offre d'emploi est importante et les salaires élevés. 
nordiques y répondent, notamment les langues scandinaves continentales. Nous ne pouvons pas rendre compte de ce vaste champ de recherche, mais vous invitons à consulter entre autres le travail important de Delsing et Lundin Åkesson?

Dans cet article, nous poursuivrons l'idée que, bien que le Norden puisse être considéré comme une communauté linguistique, il existe, notamment dans les anciennes colonies et dans les territoires actuellement non autonomes, des tensions qui ne sont pas évoquées dans les discours officiels sur l'homogénéité nordique. Nous allons traiter cette question sous la rubrique de l'hégémonie des langues dans le Norden, ou selon un terme plus positif, l'écologie des langues.

\section{ÉCOlOgIE DES LANGUES DANS LE NORDEN}

Parmi les conflits linguistiques " mineurs » (même si ce qualificatif reste à nuancer, aucun d'entre eux n'étant moindre qu'un autre), deux situations peuvent à présent être évoquées : la première concerne la tension entre le finnois et le finlandssvenska en Finlande, et la seconde la concurrence entre le nynorsk et le bokmål en Norvège. Dans les deux cas, il s'agit de pays avec un passé colonial, où la langue du pouvoir ex-colonial est impliquée dans le conflit (respectivement le suédois et le danois). Nous évoquons ces dissensions dès maintenant, car elles rompent quelque peu avec l'image idéalisée de la communauté nordique homogène. Nous allons les intégrer dans cette seconde partie où nous nuancerons l'image assez idyllique d'une communauté linguistique nordique.

Considérons l'argumentaire du Centrum för Skandinaviska studier (CSS, Copenhague/Lund) pour son colloque Rethinking Scandinavia ${ }^{10}$, tenu à l'université de Lund du 14 au 17 juin 2017 :

Au lieu d'adopter l'idée traditionnelle du Norden comme aire géographique où l'on retrouve une identité linguistique et culturelle homogène, nous considérons plutôt le Norden comme une région frontalière, où différentes langues et cultures se recoupent et se heurtent les unes aux autres, et dans laquelle de nombreux problèmes politiques brûlants arrivent à leur point critique. ${ }^{11}$

À cet égard, nous pouvons également évoquer le projet Rádstefna um tungumál og menningu á Vestur-Norðurlöndum (Contacts de langues et de cultures dans le Norden de l'Ouest), établi à Stofnun Vigdísar Finnbogadóttur (Institut Vigdís Finnbogadóttir) en Islande et dirigé par Auður Hauksdóttir. Selon lui, " les contacts de langues et de cultures dans le Norden font de cette région un véritable laboratoire linguistique ${ }^{12}$. Dans le même esprit, un autre projet,

9 Lars-Olof Delsing, Katarina Lundin Åkesson, Håller språket ihop Norden ? En forskningsrapport om ungdomars förståelse av danska, svenska och norska, Copenhague, Nordiska ministerrådet, 2005.

10 Voir <http://csspublications.net/css-conference/\#keynote>.

11 C'est l'auteur qui traduit et qui souligne.

12 Communication personnelle, Auður Hauksdóttir, septembre 2016. Voir également Helge Sandøy, " Frå tre dialektar til tre språk », in Vestnordisk språkkontakt gjennom 1200 år, G. Akselberg et E. Bugge (dir.), Tórshavn, Fróðskapur - Faroe University Press, 2011, p. 19-38. 
intitulé L'imaginaire du Nord, de l'hiver et de l'Arctique, est dirigé par Daniel Chartier, de l'université du Québec à Montréal ${ }^{13}$. Il en décrit ainsi le profil :

... une conception circumpolaire et non plus territoriale du monde froid, posant ce dernier comme un tout qui appelle des solutions, des réflexions et des positions communes, tout en tenant compte des différentes cultures et langues qui le composent, de manière plurinationale, plurilingue, pluriculturelle et, souvent, conflictuelle. ${ }^{14}$

L'argumentaire de Chartier concorde avec le nôtre, mais le transgresse en englobant toute la région circumpolaire arctique. Un pays arctique peut être défini comme un pays qui se trouve totalement ou partiellement au-delà de la limite des arbres. Selon cette définition, la région arctique comprendrait le Norden (sauf le Danemark), la Russie, l'Alaska (États-Unis) et le Canada. Cette région présente une parenté géographique et climatologique, mais aussi historique : au Moyen Âge, l'Amérique du Nord et la Russie étaient des destinations importantes des conquêtes vikings.

Pour un aperçu de la région circumpolaire arctique, considérez la carte ci-dessous :

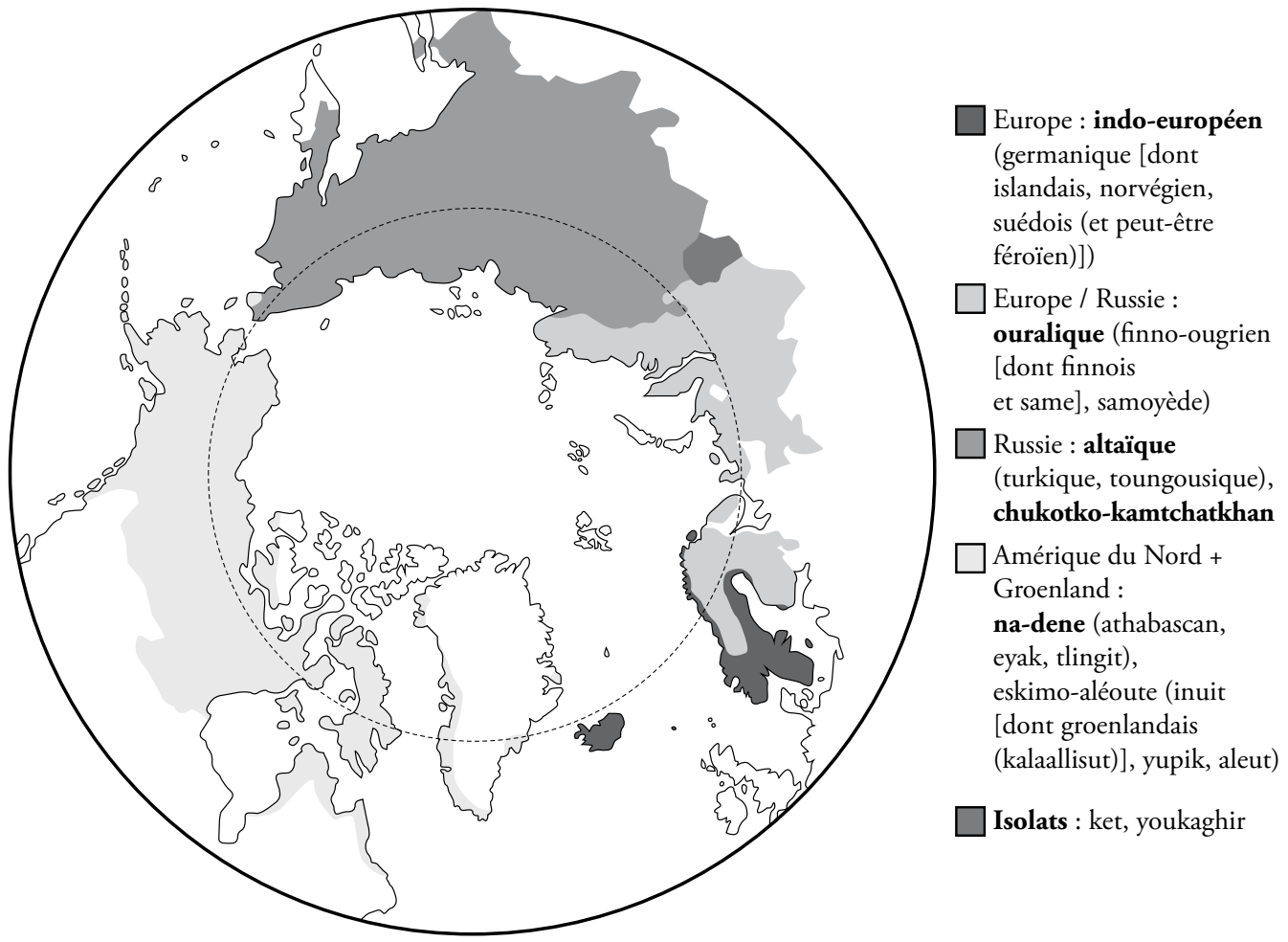

Image 1. Familles et sous-familles des langues circumpolaires

13 Voir www.imaginairedunord.uqam.ca/index.php?section=cherch_directeur.

14 C'est l'auteur qui souligne. 
L'approche de Chartier et de son équipe nous intéresse, car elle nous permet d'élargir le concept de Norden en l'incluant dans tout l'Arctique, zone dans laquelle le Norden est un acteur important. Partout dans cette aire géographique, on observe un «nouveau régionalisme » qui remplace les États-nations traditionnels - il a été évoqué un " éveil ethnique " qui transgresse les frontières traditionnelles. En 2007, les Nations unies ont fait passer une déclaration sur les droits des peuples autochtones, auxquels plusieurs communautés arctiques appartiennent. L'université de Tromsø (Norvège), la plus septentrionale au monde, héberge Arktiska rådet (Conseil arctique ; voir Nordiska rådet mentionné ci-dessus).

\section{Contacts Des Langues}

Dans des situations de contacts des langues, il est rare que les locuteurs continuent à parler leurs langues respectives sans être "impactés " par ces nouvelles rencontres linguistiques.

Au contraire, on assiste souvent aux scénarios où les langues ou les comportements des locuteurs sont complexifiés d'une manière ou d'une autre. En effet, nous pouvons dresser une typologie des aboutissements des contacts de langues dans la liste suivante, inspirée par Donald Winford ${ }^{15}$ :

- rien ne se passe, les locuteurs de la langue A continuent à parler cette langue sans apprendre B, et vice versa. Situation probablement rare, mais se reporter ici aux minorités linguistiques où des personnes bilingues spécifiques assument une fonction de " relais»;

- bilinguisme équilibré : tous les locuteurs dans un territoire donné maîtrisent $\mathrm{A}$ aussi bien que B (et inversement). Situation probablement rare, en tout cas au niveau collectif, car normalement des différences fonctionnelles se manifestent entre les deux langues impliquées (diglossie). Voir aussi le concept de near-native competence (" compétence quasi-native »);

- bilinguisme imparfait : les locuteurs de la langue A atteignent des compétences variées en langue B (qui peuvent ou ne peuvent pas apprendre A). Transfert de traits phonologiques et morphosyntaxiques, "emprunts structuraux ". Voir aussi le concept controversé de "semi-linguisme $»^{16}$ : compétence réduite dans la langue maternelle en raison de sa stigmatisation en même temps que l'accès à la langue de prestige est limité ;

- diglossie : bilinguisme, mais divergence fonctionnelle entre les deux langues impliquées. Voir le créole vs le français dans les DOM-TOM ;

- changement de langue (language shift) : la disparition d'une langue, l'adoption d'une autre. Phases d'attrition de la langue en voie de disparition : $1:$ emprunts lexicaux, 2 : interférence morphosyntaxique et phonologique, 3 : hésitation,

15 Donald Winford, An Introduction to Contact Linguistics, Oxford, Blackwell, 2003, p. 11-22.

16 Voir Nils Erik Hansegård, Tvåspråkighet eller halvspråkighet?, Stockholm, Bonniers, 1968. 
uniformisation stylistique, production formulaïque, 4 : la langue cesse d'être transmise à la nouvelle génération, mais il existe des language rememberers (" personnes qui se souviennent de la langue en question »), 5 : disparition complète de la langue. Ce processus est habituel à partir de la $2^{\mathrm{e}}$ génération parmi les locuteurs immigrés parlant des " langues d'héritage ». Il devient alors problématique de décider si ces personnes appartiennent ou non à la communauté linguistique en question :

- alternance codique / de codes (entre phrases) : fréquente parmi les locuteurs bilingues, utilisée pour des raisons diverses et complexes, stigmatisée à un certain degré. La question s'est posée de savoir si une variété caractérisée par l'alternance codique peut constituer une langue maternelle ;

- mixage des codes : changement de langue à l'intérieur de la phrase, qui peut également comprendre l'usage des mots d'emprunt non intégrés. L'alternance codique, le mixage et l'emprunt sont difficiles à délimiter clairement l'un de l'autre ; - pidginisation : émergence d'une deuxième langue réduite servant à mener à bien des transactions communicatives de base par des locuteurs qui, autrement, parlent leurs L1 respectives, comme le pidgin anglo-chinois au XVIII siècle ;

- créolisation : nativisation d'un pidgin. Définition classique, qui pourtant devient problématique quand un pidgin s'élabore (Tok Pisin, Nigerian pidgin, le français populaire d'Abidjan et éventuellement le camfranglais au Cameroun). La langue devient maternelle ou primaire pour une communauté donnée ;

- semi-créolisation : le résultat de la décréolisation d'une langue créole ou d'une créolisation partielle (" créoloïd »), voir l'afrikaans et possiblement " les langues des banlieues ";

- koïnèisation : "compromis " entre deux langues ou deux dialectes apparentés. Voir la discussion ci-dessus concernant le swahili, français québécois et «scandinave ";

- relexification : remplacement du lexique de la langue A par celui de la langue B ; - regrammaticalisation : remplacement de la morphosyntaxe de la langue A par celle de la langue B ;

- langues mixtes (language intertwining) : parfois même chose que la relexification, parfois plus complexe : en michif (français + cree, langue autotochtone du Canada), les syntagmes nominaux sont en français, mais les syntagmes verbaux en cree (parce que le michif est une langue polysynthétique, c'est-à-dire que le verbe est un morphème lexical lié, qui ne peut pas être emprunté séparément). Voir aussi media lengua (quechua + espagnol), certaines variétés de romani, etc.

Nous prenons comme point de départ l'idée selon laquelle les contacts des langues dans le Norden ont abouti aux situations qui s'inscrivent dans une ou plusieurs rubriques dans cette liste, notamment bilinguisme, diglossie, changement de langue et koïnèisation. 


\section{SURVOL HISTORIQUE}

Parmi les pays constituant le Norden, le Danemark et la Suède représentent les deux anciens pouvoirs coloniaux, tandis que la Finlande, l'Islande, et la Norvège sont considérées comme des territoires occupés maintenant indépendants, contrairement aux îles Féroé, au Sápmi, et au Groenland qui demeurent des territoires non autonomes.

Le Danemark peut être considéré comme ayant eu l'influence la plus importante lors de sa période coloniale : le pays a par exemple dominé l'ouest du Norden (les îles Orcades, Shetland, l'île de Man ainsi que certaines parties de l'Écosse et de l'Angleterre du Nord), mais comptait également des colonies aux Caraïbes (îles Vierges), en Afrique (Côte-de-l'Or dans la golfe de Guinée en Afrique) et même des comptoirs en Inde.

La Suède a plutôt contrôlé la Scandinavie continentale - à part la Finlande et la Norvège, elle avait à l'époque plusieurs dominions, notamment (i) dans la région baltique : Estonie, Ingrie, Kexholm, Livonie, Riga, Saaremaa (Ösel), et (ii) dans l'espace de l'Allemagne actuelle : Brême-et-Verden, Poméranie, Wismar.

La Suède a également connu de brèves aventures colonisatrices hors Europe, à savoir (i) en Amérique du Nord (Nouvelle-Suède), (ii) dans les Caraïbes (SaintBarthélemy et Guadeloupe), et (iii) dans la golfe de Guinée en Afrique (Côte-del'Or).

Le danois et le suédois ayant fonctionné comme langues nationales et officielles à travers les siècles, nous porterons ensuite notre attention sur les autres territoires du Norden.

\section{SURVOL GÉOPOLITIQUE DU NORDEN CONTEMPORAIN}

Les pays autonomes du Norden peuvent être catégorisés de plusieurs façons.

On distingue d'abord les anciennes colonies suédoises : (i) la Finlande, y compris Åland (actuellement avec le statut dit självstyrelse [autonomie] vis-à-vis de la Finlande), et (ii) les pays baltes, notamment l'Estonie. Or il est difficile de savoir si les pays baltes doivent être rangés parmi les pays nordiques. Il semble que l'Estonie fait des efforts considérables pour se rapprocher de l'image nordique, ce qui à un moindre degré est également le cas de la Lettonie et de la Lituanie. Il doit être souligné à cet égard que l'estonien est une langue finno-ougrienne, ce qui renforce le profil nordique du pays.

Si l'on s'intéresse maintenant au cas danois, l'Islande et la Norvège (y compris Svalbard [Spitzberg] et Jan Mayen) peuvent être considérées comme d'anciennes colonies du Danemark.

Concernant les territoires nordiques non autonomes, notons les cas suivants : (i) îles Féroé, qui jouissent du statut de heimastýrislóg / hjemmestyre (autonomie) sous le royaume danois depuis 1948, (ii) Groenland (hjemmestyre 1979), 
Selvstyreloven (loi de l'Autonomie) en 2009 vis-à-vis du Danemark, (iii) Sápmi, les territoires sames dans le nord de la Norvège, Suède, Finlande et Russie. Sápmi bénéficie d'une reconnaissance officielle limitée, mais on peut constater une certaine revitalisation linguistique (et politique ?) récente ${ }^{17}$. Le same est officiellement reconnu (signature et ratification) par la Norvège, la Suède et la Finlande dans la Charte européenne des langues régionales ou minoritaires, lancée par le Conseil de l'Europe en 1992. La Russie l’a signée sans la ratifier, mais les vingt et une républiques de la fédération de Russie ont deux langues officielles, dont le russe. Or le same n'est deuxième langue nulle part en Russie ${ }^{18}$. Ajoutons (iv) les îles Orcades, Shetland, l'île de Man, l'Écosse et l'Angleterre du Nord, où le $\dagger$ †orne se parlait à l'époque, mais qui ne constituent pas aujourd'hui des territoires nordiques et font partie du Royaume-Uni.

En plus de cette classification qui est fondée historiquement, une seconde peut être opérée à partir du critère ethnique. Distinguons d'abord les pays hébergeant une population ethnique liée au pouvoir (ex-) colonial $^{19}$, notamment (i) la Finlande (les Suédo-finlandais [finlandssvenskar] et marginalement (ii) les pays baltes [estlandssvenskar]).

Mettons ensuite en relief les pays en principe sans population ethnique liée au pouvoir (ex-)colonial : (i) Islande, (ii) Norvège, (iii) îles Féroé (?), (iv) Groenland ( ? ${ }^{20}$.

Nous pouvons également effectuer une classification par typologie linguistique $^{21}$, qui divisera les pays nordiques en deux grands groupes, d'abord (i) les pays de langue nationale germanique : Islande, Norvège, îles Féroé (pays baltes), puis (ii) les pays de langue nationale non germanique : Finlande, Groenland, Sápmi.

Finalement, nous pouvons envisager une catégorisation en termes de territoires où sont parlées de façon " exogène » les différentes langues nordiques. Cette classification nous informe que (i) le danois est parlé aux îles Féroé, en Islande, au Groenland $^{22}$, (ii) le suédois en Finlande, et auparavant, en Estonie (iii), le norvégien, le suédois, le finnois et le russe en Sápmi, puis (iv) le norne, aujourd'hui disparu, aux îles Shetland, Orcades, Man et en Écosse.

17 Voir Nordiques, no 30, 2015 : «Un autre Nord : paysages, territoires et écologie du pays same ", dossier coordonné par Karl Erland Gadelii et Marie Roué.

18 Voir le site Aménagement linguistique dans le monde, université Laval, Québec, <http://www.axl. cefan.ulaval.ca/europe/russieacc.htm>.

19 Dans les conclusions, nous allons proposer que l'émancipation linguistique est plus facile s'il n'existe pas de communauté linguistique (ex-)coloniale sur le territoire concerné.

20 Les points d'interrogation indiquent qu'il est difficile de savoir s'il existe des communautés de «Danois ethniques» au Groenland et aux îles Féroé, mais si c'est le cas, elles ne sont pas importantes.

21 L'idée étant que si la langue " endogène " est non germanique, cela faciliterait l'émancipation par rapport à la langue (ex-)coloniale.

22 Nous négligeons ici le fait qu'il était parlé en Norvège lors de sa colonisation par le Danemark. 
Des classements supplémentaires peuvent être envisagés : ceux fondés sur des facteurs géographiques, démographiques, ou encore économiques. Dans nos conclusions, nous verrons quels critères peuvent le mieux expliquer l'aboutissement de chaque situation de contacts des langues dans le Norden.

\section{DESCRIPTION DES DIFFÉRENTES (EX-)COLONIES NORDIQUES}

\section{Groenland}

Comptant de nos jours une population de 57000 habitants (peuplement majoritairement inuit), le Groenland a reçu des influences diverses tout au long de son histoire (une préhistoire amérindienne, mais une colonisation scandinave). Le territoire entre sous domination danoise en 1721 et connaît une ségrégation linguistique : le danois était la langue administrative, mais le groenlandais était parlé dans les écoles et à l'église. D'abord, il n'y avait pas d'efforts pour «danifier » les Groenlandais ${ }^{23}$ (voir le contraste bien connu entre l'idéologie coloniale de l'Angleterre [laisser faire] vs celle de la France [mission civilisatrice]), mais en 1953, le Groenland change de statut de colonie vers un amt (département) danois, avec une subséquente danification du système scolaire. Les tendances émancipatoires se renforcent cependant à partir de hjemmestyre en 1979, où le groenlandais redevient la langue de l'école. Aujourd'hui, on observe une autonomie notable vis-à-vis du Danemark dans tous les domaines, sauf dans les affaires étrangères, la défense et la monnaie. Le Groenland est le seul territoire européen à avoir quitté l'UE (en 1985), mais sa relation avec elle demeure un sujet de controverse ${ }^{24}$. Depuis 2000, on remarque une nette " groenlandisation » dans divers secteurs de la société, y compris le système éducatif ${ }^{25}$, mais le danois demeure important dans l'enseignement supérieur ${ }^{26}$. Les cadres venant du Danemark peuvent demeurer monolingues en danois (mais peut-être pas leurs enfants) ${ }^{27}$. La situation est comparable à celle des DOM-TOM et de la France, sauf que le mouvement indépendantiste est beaucoup plus fort au Groenland.

La Selvstyreloven a été adoptée en 2009 et, en mars 2013, les élections se sont soldées par la victoire des partis prônant l'indépendance. Le gouvernement actuel est composé des partis Siumut (En avant; social-démocrate rural), Inuit Ataqatigiit (Communauté inuite ; socialiste-écologiste urbain) et Naleraq (centriste nationaliste). Serait-ce le premier pas vers une indépendance totale imminente ?

23 Lars Vikør, The Nordic Languages. Their Status and Interrelations, Oslo, Novus Press, 2001, p. 111-113.

24 Le Monde, 19/10/2016.

25 Carl-Christian Olsen, "Kalaallisut - grønlandsk ", in Nordens sprog med rodder og fodder, I. S. Sletten (dir.), Copenhague, Nordisk Ministerråd, 2004, p. 126.

26 Katti Frederiksen, directeur de Greenland Secretariat, communication personnelle, septembre 2016.

27 Lars S. Vikør, The Nordic Languages. Their Status and Interrelations, $3^{e}$ éd., Oslo, Novus Press, 2001. 
Le 27 octobre dernier, un ministère chargé de l'Indépendance a été créé. Le Groenland connaîtra-t-il la même l'évolution que l'Islande?

Trond Trosterud ${ }^{28}$ soutient qu'au Groenland, on retrouve des Danois monolingues (15\%), des Groenlandais monolingues (70\%) et des Groenlandais bilingues (15\%).

Pronostic : le groenlandais s'imposera au fur et à mesure que l'indépendance se met en place, et le danois s'affaiblira (voir l'Islande). Le Groenland s'orientera peut-être plutôt vers l'Amérique du Nord que vers le Danemark, pour des raisons ethniques, culturelles et géographiques. L'indépendance de la province du Nunavut au Canada a apparemment beaucoup inspiré les indépendantistes groenlandais.

\section{Islande}

L'Islande compte aujourd'hui une population de 325000 habitants. La première colonisation de l'île par des moines irlandais date du VIII e siècle. Ensuite, on note l'arrivée des Scandinaves venant du Vestlandet, parfois via l'Irlande d'où ils ont emporté des esclaves irlandais. Le Danemark prend ensuite la relève et contrôle l'Islande jusqu'à son indépendance en 1944.

Peut-être grâce à la littérature des sagas et à la fierté du peuple islandais (comme l'Euro 2016), le danois n'a curieusement jamais réussi à s'imposer en Islande, mais en raison de sa présence dans le système éducatif islandais, les Islandais le maîtrisent et le parlent quand ils communiquent avec la Scandinavie continentale, en tout cas jusqu'à maintenant. Il est plus facile pour les Norvégiens et les Suédois de comprendre le danois parlé avec un accent islandais que de comprendre le danois parlé par les Danois ${ }^{29}$.

Selon Auður Hauksdóttir, directeur de l'Institut Vigdís Finnbogadóttir (voir ci-dessus), les Islandais apprennent le danois, mais ils se sentent plus proches des Norvégiens, entre autres parce qu'ils ont vécu tous les deux la domination danoise ${ }^{30}$.

Le danois est donc envers et contre tout invisible et inaudible en Islande, et cela ne risque pas d'évoluer puisque les jeunes générations n'aiment pas s'exprimer dans cette langue. En 2016, une enquête réalisée (non publiée) met en lumière que les adolescents islandais reconnaissent leur lien historique avec le Danemark, que certains ont des relations familiales dans ce pays, et que les jeunes Islandais

28 Trond Trosterud, "Grønlandsk, samiske språk og den nordiske språkdeklarasjonen ", Sprog $i$ Norden, 2015, p. 131-140.

29 Voir Lars-Olof Delsing, Katarina Lundin Åkesson, op. cit.

30 Auður Hauksdóttir, "'Yderst mod Norden lyser en ø...' Strejflys over islændingenes møde med dansk og norsk sprog og kultur ", in Vestnordisk språkkontakt gjennom 1200 år, op. cit., p. 39-78. 
trouvent que le Danemark est une destination pertinente pour étudier ou travailler. L'Amérique du Nord attire également les Islandais, mais ils estiment la Scandinavie plus abordable et mentalement plus proche. Quand nous avons remarqué la notable absence de la langue danoise en Islande malgré ces attitudes relativement positives, les étudiants ont rétorqué que « cela a quelque chose à voir avec notre nationalisme ".

Pronostic : la position du danois s'affaiblira encore, et connaîtra peut-être le même sort que le suédois en Finlande (tvångssvenska, "le suédois de force "), surtout comme celui-là n'a pas de base ethnique en Islande (à l'opposé du suédois en Finlande). Jusqu'à présent, les Islandais ont " regardé vers l'est " pour étudier et travailler $^{31}$. Celui-ci peut s'inverser, notamment en raison de l'influence massive de l'Amérique du Nord. Il ne s'agit plus de la présence des militaires américains sur le sol islandais comme ce fut le cas pendant la seconde guerre mondiale et la guerre froide - à l'heure actuelle, ce sont les touristes de l'Amérique du Nord qui envahissent l'île, grâce à une politique réussie de l'Islande pour promouvoir l'exotisme de son pays et en faire une escale logique pour les personnes voyageant vers ou depuis l'Amérique du Nord. Une telle évolution affaiblirait encore le danois et renforcerait l'anglais.

\section{Iles Féroé}

Avec une population de 49000 d'habitants, les îles Féroés ont été sous le contrôle danois depuis le Moyen Âge et ont obtenu le statut de hjemmestyre (heimastýrislóg) en 1948. Nous avons déjà évoqué le bilinguisme étonnant des Féroïens. Depuis des siècles, les contacts entre les îles Féroé et le Danemark restent très étroits. Beaucoup de Féroïens séjournent au Danemark pour y étudier ou travailler. Selon Karoline Küh ${ }^{32}$, le heimastýrislóg de 1948 a « dopé » la langue féroïenne, qui était d'ailleurs déjà complètement normée et standardisée. Entre autres, son orthographe avait été établie par le prêtre V. U. Hammershaimb en 1846. Une partie importante de l'enseignement se déroule en danois, et le matériel scolaire aux îles Féroé provient souvent de cette langue. Arne Torp ${ }^{33}$ observe que les Féroïens utilisent une variante du danois proche du standard quand ils communiquent avec les Danois, et dans les autres contextes scandinaves le " gøtudansk ", c'est-à-dire le danois prononcé à la féroïenne (voir le danois islandais). Les Féroïens ont des compétences très développées en danois - il est apparemment difficile de vivre aux îles Féroé sans maîtriser cette langue ${ }^{34}$, ce qui ne veut aucunement dire que le féroïen soit menacé, bien au contraire. Les îles Féroé ne

31 Carsten Jensen, Dagens Nyheter, 27/10/2016.

32 Karoline Kühl, « Danske aflæggere i Nordeuropa: Færødansk, sydslesvigdansk og bokmål », Danske talesprog, 14, 2014, p. 29-54.

33 Arne Torp, Nordiske språk i nordisk og germansk perspektiv, Oslo, Novus forlag, 1998, p. 60.

34 Ibid. 
présentent pas vraiment de communauté ethnique danoise, ce qui pourrait favoriser le statut du féroïen. Néanmoins, le bilinguisme presque parfait aux îles Féroé reste difficile à expliquer.

Pronostic : le bilinguisme férö̈en-danois se maintiendra.

4. Îles Shetland (22 000 habitants), Orcades (20 000), île de Man (86 000) et Écosse (5,3 millions)

Ces régions étaient sous dominance scandinave jusqu'au XV siècle. La langue parlée y était une variante du norrois appelée le norne, disparu autour de 1850 . Elle cohabitait avec le gaélique, aujourd'hui lui-même en péril. L'anglais s'impose actuellement, et il n'y a plus de liens linguistiques avec la Scandinavie. Toutefois, il existe une certaine affinité entre ces territoires britanniques et le Norden, qui sera vraisemblablement accentuée avec l'application du Brexit. Lors du référendum de 2014 sur l'indépendance de l'Écosse, les indépendantistes ont clamé leur différence vis-à-vis de l'Angleterre en affirmant : "Nous sommes un pays nordique. "

Pronostic : le norne est mort, le gaélique est moribond, l'anglais s'impose.

\section{Norvège}

La Norvège est aujourd'hui peuplée par 5,2 millions d'habitants. Sous contrôle danois pendant des siècles jusqu'à la rédaction de sa Constitution en 1814, elle sera rattachée à la Suède avant d'être totalement indépendante en 1905. Elle compte une minorité same, ayant le statut officiel de minorité nationale. Le pays possède deux normes écrites : le bokmål apparenté au danois et le nynorsk fondé sur les dialectes endogènes. Le nynorsk est moins utilisé que le bokmål.

Le nynorsk est une " koinè " norvégienne écrite, qui se distingue des dialectes, dont certains sont assez loin du nynorsk. Karoline Kühl ${ }^{35}$ prétend qu'en Norvège, un vrai language shift vers le danois a eu lieu lors de la prise du pouvoir du territoire par les Danois : le norgedansk était une variante du danois avec des locuteurs monolingues, à l'opposé du farødansk et du sydslesvigdansk, qui sont des versions du danois dans une situation de bilinguisme stable (farødansk + féroïen ; sydslesvigdansk + allemand). À partir de 1905, le norgedansk a disparu au bénéfice du norvégien "ethnique ". Les dialectes endogènes de la Norvège se parlaient constamment en parallèle ${ }^{36}$.

Pronostic : pas pertinent, mais le rapport de forces entre bokmål et nynorsk reste une affaire à suivre.

35 Karoline Kühl, op. cit.

36 Voir aussi Helge Sandøy, «Idéhistoria om norsk språk ", à paraître dans Norsk språkhistorie, vol. III, 2018. 


\section{Finlande}

La Finlande compte 5,5 millions d'habitants dont 300000 finlandssvenskar. La présence suédoise est millénaire, mais on retrouve également une population same dans le Nord. Les communautés suédophones ethniques se sont établies principalement dans l'archipel de Åland, dans les régions de l'Österbotten et du Nyland. Le suédois est en recul constant, mais (jusqu’à maintenant ?) bien protégé juridiquement. La législation demeure complexe et se distingue selon les municipalités monolingues finnoises ou suédoises, bilingues avec majorité finnophone ou suédophone.

Le suédois est obligatoire à l'école (mais parfois appelé tvångssvenska, "le suédois forcé »). Le " miracle finnois ", c'est-à-dire le lancement très réussi du finnois comme langue nationale, couplé à la position supérieure de la Finlande dans les enquêtes du type Pisa mesurant les connaissances des écoliers, rend critique la position du suédois. Les finlandssvenskar apprennent le finnois, mais l'inverse n'est pas aussi observable. L'usage du suédois diminue et tend à être mal vu dans beaucoup de situations. Les finlandssvenskar n'osent souvent pas demander d'être servis en suédois ${ }^{37}$. Voir aussi la montée des partis politiques du type Sannfinländarna (Les vrais Finlandais) dont le but est de complètement " fenniser » la Finlande.

Pronostic : le suédois en Finlande continuera à diminuer, le finnois s'impose.

\section{Sápmi}

Près de 80000 individus (au maximum) peuvent être considérés comme des Sames (voir définition et autodéfinition d'un same complexe). Les Sames ont obtenu la reconnaissance en tant que minorité linguistique en Norvège, Suède, Finlande, mais pas en Russie. On observe une situation de bilinguisme important, où la langue nationale prévaut. En raison des politiques assimilationnistes, le same a été abandonné et certaines variantes sont actuellement éteintes. Cependant, les tendances récentes semblent indiquer une certaine revitalisation du same du Nord et du Sud ${ }^{38}$.

Pronostic : assez négatif, mais certains signes encourageants.

\section{Pays baltes, notamment l'Estonie (1,3 millions, dont 350000 Russes)}

Vestiges du suédois, estlandssvenska. L'Estonie cultive l'image d'un pays nordique plutôt que balto-slave.

Pronostic : l'estonien s'impose, avec une présence importante du russe.

37 Språkbarometern, septembre 2016.

38 Voir Karl Erland Gadelii et Marie Roué, op. cit. 


\section{DisCUSSION}

Pour conclure, nous inscrirons ces scénarios nordiques en suivant les typologies des contacts de langues esquissées ci-dessus.

Nous considérons les quatre cas suivants comme des exemples de bilinguisme imparfait, mais de nature différente :

\section{Bilinguisme imparfait :}

- Groenland : le danois s'affaiblit, le groenlandais (kalaallisut) s'impose ;

- Islande : le danois s'affaiblit, l'islandais s'impose. Voir l'enquête de terrain mentionnée ci-dessus, que l'auteur a effectuée parmi des étudiants en Islande en 2016. Cette enquête montre que, si les Islandais " regardent toujours vers l'est ", ils considèrent la langue danoise comme peu utile, y recourent rarement et, par conséquent, la parlent mal. Le scénario ici est plus clair que dans le cas du Groenland, entre autres parce que l'Islande est un État autonome ;

- Finlande : le suédois s'affaiblit, le finnois s'impose. Mais ici la situation est encore différente des deux premières, car à l'opposé du Groenland et de l'Islande, la Finlande héberge une minorité ethnique parlant la langue ex-coloniale, les finlandssvenskar. La situation est compliquée, car les Suédo-finnois sont en principe obligés d'être bilingues, mais l'étiquette " bilinguisme équilibré " paraît un peu étrange dans leur cas, vu qu'ils sont normalement d'ardents défenseurs de la langue suédoise. Les Finnois, de leur côté, peuvent très bien être monolingues en finnois, bien que le pays soit officiellement bilingue. Donc dans leur cas, «bilinguisme imparfait » est sans doute une expression trop positive ;

- Sápmi : le same s'affaiblit tandis que le norvégien, le suédois, le finnois et le russe s'imposent. À l'opposé des trois cas de figure précédents, la langue affaiblie ici n'est pas la langue (ex-)coloniale, mais la langue endogène, le same. Si le same risque de disparaître, nous avons plutôt un cas de language shift comparable au dernier scénario ci-dessous, où l'anglais prend le pas sur le gaélique et le norne.

\section{Bilinguisme équilibré :}

- Îles Féroé : féroïen + danois.

\section{Language shift :}

- Norvège : danois (norgedansk) $\rightarrow$ norvégien;

- Estonie : suédois (entre autres) $\rightarrow$ estonien ;

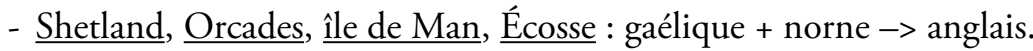

Dans les deux premiers cas, c'est la langue endogène qui est reconquise, tandis que dans le dernier cas, c'est une langue exogène, l'anglais, qui s'établit comme la cible du changement de langue. 
Nous sommes d'avis que les trois cas de language shift sont assez clairs : dans l'exemple de la Norvège et de l'Estonie, l'ancien pouvoir colonial a disparu, et dans le dernier un nouveau pouvoir « colonial » est arrivé. Les citoyens parlent donc la langue du pouvoir politique.

Les cas de bilinguisme imparfait et équilibré sont plus difficiles à analyser. La situation au Sápmi ressemble à celle de Shetland où une petite langue endogène fléchit devant la langue du pouvoir. Les samophones sont particulièrement vulnérables, dans la mesure où ils n'occupent pas de territoire bien défini. En effet, ils sont dispersés dans quatre pays différents sans frontières nettes. En ce qui concerne la Finlande, nous avons déjà noté que le finnois tout comme le suédois sont des langues officielles, ce qui signifie que le suédois n'est pas techniquement en péril tel le same. Mais à terme, nous pourrions spéculer sur la possibilité d'un language shift parmi les finlandssvenskar (ou un exode massif vers la Suède) du même type que les Sames sont peut-être en train de vivre actuellement.

Restent alors trois territoires : le Groenland, l'Islande et les îles Féroé. Comme indiqué ci-dessus, le bilinguisme imparfait en Islande peut facilement s'expliquer par le fait que ce pays est devenu indépendant en 1945, ce qui a officialisé le statut de l'islandais, une langue pérennisée à l'écrit grâce à sa longue production. Ce développement a considérablement affaibli la position du danois.

Il ne subsiste alors qu'une énigme à résoudre : pourquoi le Groenland se caractérise-t-il par un bilinguisme imparfait (ou, en d'autres termes, par une langue endogène dominante) et les îles Féroé par un bilinguisme équilibré ? Superficiellement, il s'agit de deux territoires qui se ressemblent : ils sont insulaires, non autonomes, à faible population, avec une langue nationale parlée par $100 \%$ de la population, et sans communauté linguistique coloniale sur le territoire. Nous sommes d'avis que trois facteurs peuvent expliquer la différence entre le Groenland et les îles Féroé :

- la simple distance géographique : le Groenland est beaucoup plus éloigné du Danemark que les îles Féroé -> la distance favorise la langue endogène ;

- le facteur linguistique : le groenlandais n'est pas une langue indo-européenne, tandis que le féroïen est une langue nordique à base germanique, comme le danois $\rightarrow$ la différence typologique favorise la langue endogène ;

- l'économie : le Groenland, à l'opposé des îles Féroé, est une économie émergente $\rightarrow$ l'autonomie économique favorise la langue endogène.

Ces trois facteurs semblent pouvoir expliquer pourquoi le groenlandais domine au Groenland au détriment du danois, tandis que les liens géographique, linguistique et économique entre les îles Féroé et le Danemark donnent lieu à un bilinguisme équilibré féroïen + danois. 
Mais pourquoi le féroïen n'est-il pas une langue en péril ? Nous avons vu que tous les risques sont présents : les îles Féroé sont dominées par le Danemark au niveau politique, économique, linguistique, démographique et géographique. Pourquoi les Féroïens n'ont-ils pas tout simplement adopté le danois ? Nous sommes d'avis que le cas féroïen ressemble à celui de l'islandais. Les Islandais n’ont jamais abandonné leur langue en faveur du danois, malgré une domination danoise séculaire. Selon nous, trois facteurs ont contribué à la survie de la langue islandaise : une riche culture ancestrale, une fierté nationale, et le fait de se trouver sur un territoire délimité. Les îles Féroé présentent exactement les mêmes caractéristiques, mais à l'opposé de l'Islande, elles ne sont pas autonomes. L'équilibre vient donc de la combinaison des facteurs internes (culture, fierté, territoire) et externes (non-autonomie). Le jour où les îles Féroé deviendront indépendantes, le bilinguisme équilibré va se transformer en bilinguisme imparfait : le féroïen va prendre le pas sur le danois.

Pour revenir à la question initiale - le Norden constitue-t-il une communauté linguistique ? -, le compte rendu ci-dessus indique qu'au sens étroit, cela n'est pas le cas. Or au sens large il peut l'être, étant donné que les langues des anciens pays colonisateurs, c'est-à-dire le danois et le suédois, peuvent toujours fonctionner comme linguae francae en communication intra ${ }^{39}$. Mais cette situation est en train de changer, et l'on peut envisager, suivant les modèles finnois et islandais, l'émancipation du groenlandais et du férö̈en. L'intercompréhension nordique ne serait alors plus une réalité, dans la mesure où on n'aura plus recours à une langue de la Scandinavie continentale afin d'assurer la compréhension mutuelle, et l'anglais prendra le pas, pour le meilleur et pour le pire.

39 Pour la Norvège, la question ne se pose évidemment pas. 